\title{
Comparison of spinal and cerebral oxygen saturation with near-infrared spectroscopy method during spinal surgery in prone position
}

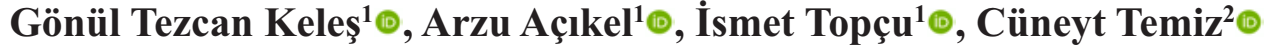 \\ ${ }^{1}$ Department of Anesthesiology and Reanimation, Celal Bayar University School of Medicine, Manisa, Turkey \\ ${ }^{2}$ Department of Neurosurgery, Celal Bayar University School of Medicine, Manisa, Turkey
}

DOI: $10.18621 /$ eurj.371777

\begin{abstract}
Objectives: To assess spinal and cerebral oxygenation with near-infrared spectroscopy method during spinal surgery in prone position.

Methods: This prospective study included 64 patients, who were prepared for posterior spinal instrumentation and laminectomy surgeries. Group 1: 31 patients who had posterior spinal instrumentation; Group 2: 33 patients who had at least two levels of disk operation (Laminectomy). The following were recorded for all patients before and after anesthesia induction in supine position, after induction in prone position, during operation (beginning, middle and end of surgery) in prone position and before waking up in supine position: cerebral oxygen saturation (NIRSs) measurements, spinal oxygen saturation (NIRSp) measurements, peripheral oxygen saturation $\left(\mathrm{SpO}_{2}\right)$, heart rate (HR), invasively monitored systolic arterial pressure (SAP), diastolic arterial pressure (DAP), mean arterial pressure (MAP) values.

Results: There was no significant difference between two groups in terms of the variables of age, weight, anesthesia and surgery duration $(p>0.05)$. MAP values were significantly different in both groups before induction, during operation and postoperative periods $(p<0.05)$. NIRSs were significantly lower at midoperation and at the end of operation $(p<0.05)$. NIRSp values had no significant difference in any period $(p$ $>0.05)$. We found no significant difference between groups in HR, SAP, DAP, MAP, NIRSs and NIRSp parameters $(p>0.05)$ compared at different times.

Conclusions: MAP dropped depending on induction and prone position. Cerebral oxygenation significantly decreased at the time of mid-operation and at the end of operation but spinal oxygenation had no significant decresase. Compared to laminectomy, posterior stabilization surgery did not pose an additional risk to the patients in terms of spinal and oxygen saturation.
\end{abstract}

Keywords: prone position, spinal surgery, cerebral oxygenation, near-infrared spectroscopy

Received: December 27, 2017; Accepted: June 21, 2018; Published Online: July 28, 2018

$\mathrm{T}$ o prevent the possible complications, it is important to recognize symptoms such as ischemia and hypoxia at an early stage which might develop during spinal surgery due to surgical intervention or pre-existing trauma. Spinal surgeries are mostly performed in prone position. Spinal surgery presents a complex

Address for correspondence: Arzu Açıkel, MD., Celal Bayar University School of Medicine, Department of Anesthesiology and Reanimation, Manisa,Turkey 
situation where surgery trauma, general anesthesia and prone position generate hemodynamic and respiratory effects. It is important to follow the changes in cerebral and spinal perfusion and oxygenation. Compared to discectomy and laminectomy, posterior stabilization surgeries pose more risk in terms of cerebral and spinal oxygen saturation due to more compression and bleeding risk.

In near-infrared spectroscopy (NIRS) method, infrared light is used to penetrate the tissue, and the optic values of oxyhemoglobin and deoxyhemoglobin in capillary bed are used to calculate the oxygen saturation in tissue [1]. It is a noninvasive method. It enables the measurement of cerebral tissue oxygen saturation with the help of the sensors placed on frontal region. Possibility of measurement mistakes prevents getting the real values for cerebral oxygen saturation and limits its use [2,3]. Many factors such as the arterial and venous vascular density of the region where sensor is applied, arterio-venous shunts, hemodilution, edema, and patient's bilirubin level might prevent finding the real oxygenation value not only in cerebral tissue but in all measured tissues [2]. However, it is advantageous in terms of the real-time monitoring of oxygenation and its trend follow-up [4, 5]. It has also been reported that, in the light of the insufficiency of standard monitoring methods in following the changes in tissue oxygenation, NIRS monitoring of spinal cord will be a useful warning system for reducing ischemic spinal cord damages, which might occur due to spinal cord surgery [6-9].

In this study, our purpose was to find out whether patients' spinal and cerebral oxygenation values varied before induction, during operation and after operation, and to assess the relation between tissue oxygenation changes and hemodynamic changes in two different survey groups which had posterior spinal instrumentation and at least two levels of disk operation (laminectomy).

\section{METHODS}

The study started after getting the Local Ethics Council Approval from the Medical School of Celal Bayar University (26/02/2014, No: 20478486-100) and CBU BAP support (project no. 2014-076).

The study was prospective and included 64 patients who were prepared by the Neurosurgery Clinic for posterior spinal instrumentation and laminectomy, ASA I, II and III (American Society of Anesthesiologists classification) between May 2014 and May 2016. The patients aged between 18 and 70 years, were included in the study after their informed consents were received. Those who did not consent to take part in the study, pregnant patients, those with pre-diagnosed vascular operation and diseases (abdominal aorta surgery, etc.) or a history of cardiac surgery or any intracranial pathology were excluded. The patients, who were included in the study for their spinal surgeries were divided into two groups based on the surgery they underwent; Group 1: 31 cases who had posterior spinal instrumentation (Group 1, $\mathrm{n}=31$ ) and Group 2: 33 cases who had at least two levels of disk operation (Laminectomy) (Group 2, $\mathrm{n}=33$ ).

All patients were monitored for heart rate (HR), invasive arterial blood pressure, peripheral oxygen saturation ( $\mathrm{SpO} 2)$, and end-tidal $\mathrm{CO} 2$ (EtCO2) after being taken into the operating room. In addition, Group 1 cases were monitored for central venous pressure (CVP). For monitoring tissue oxygenation, NIRS (NONIN-Somanetics, Nonin Medical Inc. Minnesota, $\mathrm{MN}$ ) probes were placed on frontal region and on the distal of spinal segment to be operated.

Pre-oxygenation was performed for all cases with $100 \%$ oxygen. Then, the same general anesthesia induction and anesthesia maintenance were applied to both groups. General anesthesia induction was performed with: fentanyl 1-2 $\mu \mathrm{g} / \mathrm{kg}$, propofol 2-3 $\mathrm{mg} / \mathrm{kg}$, rocuronium $0.9 \mathrm{mg} / \mathrm{kg}$. After endotracheal intubation, mechanic ventilation parameters were set to keep the tidal volume at $7 \mathrm{ml} / \mathrm{kg}$ and end-tidal $\mathrm{CO} 2$ value at $30-40 \mathrm{mmHg}$. Anesthesia maintenance was provided with sevoflurane $1-2 \%, 40 / 60 \% \mathrm{O} 2 / \mathrm{N} 2 \mathrm{O}$ mix and remifentanil infusion. After the patient was put in prone position, endotracheal tube location was verified; eyeballs, nose and other possible pressure locations were checked; and proper position preventing abdominal pressure was confirmed.

Cerebral oxygen saturation (NIRSs) was measured with the NIRS device (NONIN-Somanetics) by placing probes on the foreheads of patients in both groups; and spinal oxygen saturation (NIRSp) was measured by placing NIRS probes which were sterilized by immersing into ethylene oxide on the distal dermis of the spinal segment to be operated. 
Table 1. Descriptive demographical information of groups

\begin{tabular}{lccc}
\hline & Group 1 (n= 31) & Group 2 (n= 33) & $\boldsymbol{p}$ \\
\hline Age (years) & $53.77 \pm 13.90$ & $52.87 \pm 13.29$ & 0.690 \\
Weight (kg) & $78.70 \pm 14.56$ & $79.66 \pm 12.31$ & 0.590 \\
BMI (kg/m $\mathbf{~}^{2}$ & $26.69 \pm 6.11$ & $27.03 \pm 5.27$ & 0.610 \\
Gender (male/female) & $17 / 14$ & $19 / 11$ & \\
ASA (1/2/3) & $10 / 16 / 5$ & $11 / 15 / 7$ & \\
Anesthesia duration (min) & $154.35 \pm 38.29$ & $160.15 \pm 39.30$ & 0.710 \\
Surgery duration (min) & $137.25 \pm 38.79$ & $137.72 \pm 35.99$ & 0.680 \\
\hline
\end{tabular}

Data are shown as mean \pm standard deviation or number. Group 1 =cases who had posterior spinal instrumentation, Group 2 = cases who had at least two levels of disk operation (Laminectomy), ASA = American Society of Anesthesiologists classification, BMI = body mass index

Simultaneously; SpO2, HR, invasively monitored systolic arterial pressure (SAP), diastolic arterial pressure (DAP), mean arterial pressure (MAP) values were recorded.

Measurement times include T1: Before induction (Supine position), T2: After induction (Supine), T3: Right before surgery (after turning to prone position), T4: After starting the surgery (Prone), T5: Mid-surgery (Prone), T6: End of surgery-at the time of skin suturing (Prone), and T7: After the final positioning of the patient (Supine).

When there is an instant changing in the recorded cerebral oxygenation values the surgery teams were simultaneously warned.

\section{Statistical Analysis}

SPSS (statistical package for social sciences for Windows 15.0) program was used for the statistical analysis of the study. Data were evaluated through descriptive statistical analysis by using mean, standard deviation, minimum, maximum, interval and percentage distributions. Analytically, the two groups were evaluated by applying $\mathrm{T}$ test in independent groups. Pearson correlation test was applied for comparing numeric data. In all analytical evaluations, $p<0.05$ was the significance limit value.

\section{RESULTS}

We found no significant difference between two groups in terms of the variables of age, weight, anesthesia and surgery duration $(p>0.05)$ (Table 1$)$.
In inter-group comparisons, there was no significant difference between two groups in HR, SAP, DAP, MAP, NIRSs and NIRSp parameters compared at all times $(p>0.05)$. But, there were significant differences between the values taken different measure-times for each group of patients.

Among group 1, there was a significant difference between T1 and T2 MAP values $(p<0.001)$. T2 MAP value was significantly different from T3 MAP value $(p=0.0019)$. Among group 2, there was a significant difference between T1 and T2, T2 and T3 MAP values $(p<0.001$ and $\mathrm{p}=0.015$, respectively). Group 1 and Group 2 MAP values were given in Table 2.

Among group 1, there was a significant difference between T1 NIRSs value and T3 NIRSs value ( $p=$ $0.04)$. T3 NIRSs value was significantly different from T5 and T6 NIRSs values ( $p=0.018$ and $p=0.032$,

Table 2. Group 1 and Group 2 MAP values

\begin{tabular}{lcc}
\hline & $\begin{array}{c}\text { Group 1 } \\
(\text { mean } \pm \text { SD) }\end{array}$ & $\begin{array}{c}\text { Group 2 } \\
(\text { mean } \pm \text { SD) }\end{array}$ \\
\hline MAP T1 & $96.48 \pm 15.09$ & $97.33 \pm 14.96$ \\
MAP T2 & $79.58 \pm 12.70$ & $80.60 \pm 13.52$ \\
MAP T3 & $73.19 \pm 12.73$ & $74.39 \pm 13.96$ \\
MAP T4 & $75.16 \pm 11.85$ & $74.90 \pm 11.51$ \\
MAP T5 & $71.54 \pm 8.64$ & $72.06 \pm 8.70$ \\
MAP T6 & $76.48 \pm 15.83$ & $77.24 \pm 15.64$ \\
MAP T7 & $91.83 \pm 11.44$ & $91.87 \pm 11.07$ \\
\hline Group 1 =cases who had posterior spinal instrumentation, \\
Group 2 = cases who had at least two levels of disk operation \\
(Laminectomy), MAP $=$ mean arterial & pressure, SD $=$ \\
standarddeviation &
\end{tabular}


Table 3. Group 1 and Group 2 NIRSs values

\begin{tabular}{lcc}
\hline & $\begin{array}{c}\text { Group 1 } \\
(\text { mean } \pm \text { SD) }\end{array}$ & $\begin{array}{c}\text { Group 2 } \\
(\text { mean } \pm \text { SD) }\end{array}$ \\
\hline NIRSs T1 & $77.83 \pm 12.84$ & $77.15 \pm 12.76$ \\
NIRSs T2 & $73.80 \pm 11.42$ & $73.63 \pm 11.10$ \\
NIRSs T3 & $71.25 \pm 12.89$ & $70.84 \pm 12.65$ \\
NIRSs T4 & $69.06 \pm 9.50$ & $68.81 \pm 9.29$ \\
NIRSs T5 & $66.67 \pm 9.77$ & $66.57 \pm 9.52$ \\
NIRSs T6 & $67.06 \pm 11.75$ & $67.37 \pm 11.51$ \\
NIRSs T7 & $70.76 \pm 11.69$ & $71.00 \pm 11.15$ \\
\hline
\end{tabular}

Group $1=$ cases who had posterior spinal instrumentation, Group 2 = cases who had at least two levels of disk operation (Laminectomy), NIRSs = cerebral oxygen saturation, $\mathrm{SD}=$ standard deviation

respectively). Among group 2, there was a significant difference between T1 and T3, T3 and T5 NIRSs values ( $p=0.038$ and $p=0.021$, respectively). NIRSs values of Group 1 and Group 2 patients were detailed in Table 3.

The correlation analysis of the intra-group comparisons of Group 1 MAP and NIRSs values indicated a positively $(\mathrm{r}=0.427)$ significant $(p=0.017)$ relation between T5 MAP and T5 NIRSs, and a positively $(\mathrm{r}=0.486)$ significant $(p=0.006)$ relation between T6 MAP and T6 NIRSs (Tables 2 and 3)

The correlation analysis of the intra-group comparisons of Group 2 MAP and NIRSs values indicated a negatively $(\mathrm{r}=-0.375)$ significant $(p=$ $0.032)$ relation between T1 MAP and T1 NIRSs; a positively $(\mathrm{r}=0.383)$ significant $(p=0.028)$ relation between T5 MAP and T5 NIRSs; and a positively ( $\mathrm{r}=$ $0.504)$ significant $(p=0.003)$ relation between T6 MAP and T6 NIRSs (Tables 2 and 3).

\section{DISCUSSION}

We found that, compared to the MAP values measured before induction, MAP values dropped with anesthesia induction and significantly dropped in prone position during operation. With respect to NIRSs; while there was no difference in supineposition before and after induction, there was a decline after turning to prone position. We found a positively significant relation between MAP and NIRSs during the time of ongoing surgery. This finding was also supported by other studies in literature. The study by Babakhani et al. [4], which enrolled 50 patients undergoing lumbar spine surgery in prone position, concluded that cerebral oxygenation can be maintained in prone position and it is very crucial in preventing bradycardia and arterial hypotension. The study by Trafidlo et al. [10], which covered 43 patients, that underwent lumbar spondylosis surgery in prone position to assess postoperative cognitive functions, indicated a positive correlation between MAP and cerebraloxygensaturation. The study by Meng et al. [11] reported that this change was actually associated with cardiac output (CO) while it was affected by other hemodynamic parameters. In our study, we thought thatthe change in NIRSs actually derived from prone position while it was affected by the decline inMAP. We can say that this effect of prone position is a complex combination of many effects such as increased abdominal pressure, thoracic compression, disrupted venousturn, cardiac output return, effects on respiratory dynamics, and effects on intracranial circulation, as well as MAP. Studies reported that pulmonary infiltration and atelectatic regions, which concentrated in posterior lung in supine position, dissolved in prone position, intrapulmonary shunting decreased, and perfusion increased in ventral [12]. The clear conclusion of all these was an increased oxygenation in prone position [12]. However, the obstruction that occurred in inferior vena cava in prone position caused a decrease in cardiac venous return [13], and resulted in decreased cardiac index and cardiac output $[13,14]$. With the help of autoregulation mechanisms, systemic vascular resistance (SVR) was increased and MAP was maintained [13, 15]. However, anesthesia induction might disrupt the rise in SVR and auto-regulation thereby causing a drop in MAP [16]. When we consider the results of our study and all effects of prone position on systems, we might conclude that cerebral oxygenation might be affected negatively. The study by Babakhani et al. [4] indicated the decrease in cerebral oxygenation in prone position while it was within the margin of safety.

Compared to laminectomy, posterior stabilization operations require applying more compression for placing rots and they pose a bleeding risk. The study by Park [17], which covered 40 patients that underwent spinal surgery in prone position, reported that an increase in intraabdominal pressure caused an 
increase in blood loss in surgery region. Due to those differences, posterior stabilization operations might have a more negative effect on average arterial pressure change, cerebral and spinal oxygen saturation due to venous return and volume loss, compared to laminectomies. By including these two types of surgeries performed in prone position in our study, we aimed to compare the oxygenation values before, during and after operation in these groups. In our study, the groups had a homogenous distribution in terms of demographic data. The comparison of two groups indicated no difference in terms of hemodynamic data, NIRSs and NIRSp values. This led us to think that compared to laminectomy, stabilization surgeries did not pose an additional risk to the patient in terms of cerebral and spinal oxygen saturation values. It is possible to say that accurate surgery technic and proper bleeding intervention decreased cerebralo xygenation risk.

In our study, we saw no significant difference in NIRSp values in any period. Similar to the NIRSs values, NIRSp values had a statistically insignificant decrease in proneposition. This might have resulted from an increase in venous blood rate in the region covered by NIRSp sensor as a result of venous stasis. Moreover, NIRSp measurement might be affected by differences in skin, subcutaneous fat tissue and muscle tissue volume and blood build-up. There are studies indicating that paravertebral muscle volume changed based on age and gender [18]. However, since our study found no statistically significant difference between groups in terms of age, gender and BMI, we think that NIRSp measurements were not affected by those factorsin our study.

\section{Limitations}

The limitations of NIRS method in the other studies have also limited our study. Many factors such as the arterial and venous vascular density of the region where sensor is applied, arterio-venous shunts, hemodilution, edema, and patient's bilirubin level might have affected the real value of oxygenation.

\section{CONCLUSION}

In conclusion, MAP dropped depending on induction and prone position. Cerebral oxygenation decreased significantly at the time of mid-operation and at the end of operation but there was no significant decrease for spinal oxygenation. Compared to laminectomy, posterior stabilization surgeries did not pose an additional risk to the patient in terms of cerebral and spinal oxygensaturation.

\section{Conflict of interest}

The authors disclosed no conflict of interest during the preparation or publication of this manuscript.

\section{Acknowledgement}

This study was supported by Scientific Research Project Office of Manisa Celal Bayar University, School of Medicine, and No. 2014-076.

\section{REFERENCES}

[1] Moerman AT, De Hert SG, Jacobs TF, De Wilde LF, Wouters PF. Cerebral oxygen desaturation during beach chair position. Eur J Anaesthesiol 2012;29:82-7.

[2] Steppan J, Hogue CW Jr. Cerebral and tissue oximetry. Best Pract Res Clin Anaesthesiol 2014;28:429-39.

[3] Madsen PL, Secher NH. Near-infrared oximetry of the brain. Prog Neurobiol 1999;58:541-60.

[4] Babakhani B, Heroabadi A, Hosseinitabatabaei N, Schott M, Yekaninejad S, Jantzen JP, et al. Cerebral oxygenation under general anesthesia can be safely preserved in patients in prone position: a prospective observational study. J Neurosurg Anesthesiol 2017;29:291-7.

[5] Pollard V, Prough DS, DeMelo AE, Deyo DJ, Uchida T, Widman R. The influence of carbon dioxide and body position on near-infrared spectroscopic assessment of cerebral hemoglobin oxygen saturation. Anesth Analg 1996;82:278-87.

[6] Macnab AJ, Gagnon RE, Gagnon FA, LeBlanc JG. NIRS monitoring of brain and spinal cord detection of adverse intraoperative events. Spectroscopy 2003;17:483-90.

[7] Toraman F, Özgen SU, Aritürk C, Sayın J, Erkek E, Güçlü P, et al. [Is it efficient to use NIRS to calculate hepatic and renal oxygen saturation during extracorporeal circulation?]. Acıbadem Üniversitesi Sağlı Bilimleri Dergisi 2012;3:164-9. [Article in Turkish]

[8] Murkin JM, Arango M. Near-infrared spectroscopy as an index of brain and tissue oxygenation. Br J Anaesth 2009;103 Suppl 1:i3-13.

[9] Macnab AJ, Gagnon RE, Gagnon FA. Near infrared spectroscopy for intraoperative monitoring of the spinal cord. Spine 2002;27;17-20.

[10] Trafidło T, Gaszyński T, Gaszyński W, NowakowskaDomagała K. Intraoperative monitoring of cerebral NIRS oximetry leads to better postoperative cognitive performance: a pilot study. Int J Surg 2015;16(PtA):23-30.

[11] Meng L, Cannesson M, Alexander BS, Yu Z, Kain ZN, 
Cerussi AE, et al. Effect of phenylephrine and ephedrine bolus treatment on cerebral oxygenation in anaesthetized patients. Br J Anaesth 2011;107:209-17.

[12] Badia JR, Sala E, Rodriguez-Roisin R. Positional changes and drug interventions in acute respiratory failure. Respirology 1998;3:103-6.

[13] Edgcombe H, Carter K, Yarrow S. Anaesthesia in the prone position. Br J Anaesth 2008;100:165-83.

[14] Yokoyama M, Ueda W, Hirakawa M, Yamamoto H. Hemodynamic effect of the prone position during anesthesia. Acta Anaesthesiol Scand 1991;35:741-4.

[15] Sudheer PS, Logan SW, Ateleanu B, Hall JE. Haemodynamic effects of the prone position: a comparison of propofol total intravenous and inhalation anaesthesia. Anaesthesia 2006;61:138-41.

[16] Poon KS, Wu KC, Chen CC, Fung ST, Lau AW, Huang CC, et al. Hemodynamic changes during spinal surgery in the prone position. Acta Anaesthesiol Taiwan 2008;46:57-60.

[17] Park CK. The effect of patient positioning on intraabdominal pressure and blood loss in spinal surgery.Anesth Analg 2000;91:552-7.

[18] Ekin EE, Kurtul Yıldız H, Mutlu H. Age and sex-based distribution of lumbarmultifidus muscle atrophy and coexistence of disc hernia: an MRI study of 2028 patients. Diagn Interv Radiol 2016;22:273-6. 